\title{
Active Online Assessment of Patients Using New Oral Anticoagulants: Bleeding Risk, Compliance, and Coagulation Analysis
}

\author{
Birgitta Salmela, M.D., Ph.D. ${ }^{1} \quad$ Lotta Joutsi-Korhonen, M.D., Ph.D. ${ }^{2} \quad$ Elina Armstrong, M.D., Ph.D. ${ }^{1}$ \\ Riitta Lassila, M.D., Ph.D. ${ }^{1,2}$ \\ ${ }^{1}$ Coagulation Disorders, Division of Hematology, Department of \\ Internal Medicine, Helsinki University Central Hospital, Helsinki, \\ Finland \\ ${ }^{2}$ Clinical Chemistry, HUSLAB Laboratory Services, Helsinki University

\begin{abstract}
Address for correspondence and reprint requests Birgitta Salmela, M.D., Ph.D., Coagulation Disorders, Division of Hematology, Department of Internal Medicine, Helsinki University Central Hospital, P.O. Box 340, 00029 HUCH, Helsinki, Finland (e-mail: birgitta.salmela@hus.fi).
\end{abstract} Central Hospital, Helsinki, Finland

Semin Thromb Hemost 2012;38:23-30.

\begin{abstract}
Keywords

- bleeding risk

- new oral anticoagulants

- laboratory measuring

- dabigatran

- rivaroxaban

- apixaban

Clinicians prescribing new oral anticoagulants (OACs; dabigatran, rivaroxaban, and apixaban) should be aware of the exclusion criteria related to bleeding risks defined in published clinical studies. At least a quarter of patients currently using warfarin have an exclusion criterion that may prevent easy transition to the new OACs. In the summary of product characteristics for dabigatran, as an example, the target populations appear generalized. Due to fixed dosing and predictable pharmacology, routine laboratory monitoring of new OACs is deemed unnecessary. Under special circumstances, however, understanding the extent of thrombin or factor $(F)$ Xa inhibition may aid in evaluating compliance and handling emergency interventions, bleeding complications, or overdoses. Although commonly available global coagulation-time assessments (prothrombin time and activated partial thromboplastin time) are insensitive, they may assist clinical management by indicating a severe accumulation of OACs; moreover, a normal thrombin time (TT) excludes a thrombin-inhibitor effect. In particular circumstances, specific assays (diluted TT, Ecarin clotting time, anti-Flla or anti-FXa activity) may quantify the anticoagulant effect, but therapeutic ranges for dose adjustment are not yet established. Laboratory results are also influenced by clinical situation: e.g. bleed (consumption of coagulation factors) versus postoperative state (activation of coagulation). Without specific antidotes and evidence-based treatment strategies, new OACs are clinically worrisome in patients with impaired renal or liver function. Postmarketing surveillance and recording of bleeding complications (ICD-10 D68.32) are therefore of major importance.
\end{abstract}

One-fourth of all patients on vitamin $\mathrm{K}$ antagonist (VKA) therapy, when admitted to hospital for an infection would have been excluded from practically all clinical trials of oral anticoagulants (OACs) for atrial fibrillation (AF), for secondary prevention of venous thromboembolism (VTE), or for prevention of coronary atherothrombosis. ${ }^{1}$ Similar exclusion criteria occurred even more frequently among patients hospitalized for a bleeding complication while on VKA; at least one exclusion criterion emerged in $40 \%$ of such cases. ${ }^{1}$ It is noteworthy that six principal randomized studies for the present treatment guidelines on VKA for AF excluded 87\% of the patients screened. ${ }^{1}$ The presence of at least one
Issue Theme Hot Topics III; Guest Editor, Emmanuel J. Favaloro, Ph.D., M.A.I.M.S., F.F.Sc. (RCPA)
Copyright $\odot 2012$ by Thieme Medical Publishers, Inc., 333 Seventh Avenue, New York, NY 10001, USA. Tel: +1(212) 584-4662.
DOI http://dx.doi.org/ 10.1055/s-0031-1300948. ISSN 0094-6176. 
Table 1 Bleeding Risk-Related Exclusion Criteria in Studies Comparing New OACs with Conventional Treatment (LMWH and Vitamin K Antagonist) for VTE

\begin{tabular}{|l|l|}
\hline $\begin{array}{l}\text { Study, Mean Duration } \\
(\boldsymbol{n}=\text { Patients with New OAC) }\end{array}$ & Bleeding Risk-Related Exclusion Criteria \\
\hline $\begin{array}{l}\text { Dabigatran } \\
\text { (RE-COVER) }\end{array}$ & High risk for bleeding \\
$163 \pm 50 \mathrm{~d}$ & Contraindication to heparin \\
$(n=1273)$ & Requirement for long-term antiplatelet therapy \\
& ASA $>100 \mathrm{mg}$ \\
\hline Rivaroxaban & Active bleeding or high risk of bleeding \\
$($ Einstein-DVT) & Any other contraindication listed in the labeling of permitted anticoagulants \\
$83-87 \mathrm{~d}$ & Treatment for $>36$ h before randomization with therapeutic doses of UFH or LMWH, \\
$(n=405)$ & or receiving more than a single dose of a VKA \\
& SBP $>200$ mm Hg or DBP $>110$ mm Hg \\
\hline $\begin{array}{l}\text { Apixaban } \\
(\text { Botticelli DVT) }\end{array}$ & Active bleeding or high risk for bleeding contraindicating treatment with LMWH, \\
$80 \pm 20 \mathrm{~d}$ & fondaparinux, or VKA \\
$(n=392)$ & Any other contraindication listed in the local labeling of enoxaparin, tinzaparin, \\
& fondaparinux, warfarin, acenocoumarol, or phenprocoumon \\
& $>24$ h of prerandomization treatment with therapeutic doses of UFH, LMWH, \\
& or fondaparinux \\
& More than a single starting dose of VKA before randomization \\
& SBP $>200$ mm Hg or DBP $>110$ mm Hg \\
& ASA $>165$ mg \\
\hline
\end{tabular}

OACs, oral anticoagulants; LMWH, low-molecular-weight heparin; VKA, vitamin K antagonist; VTE, venous thromboembolism; ASA, acetylsalicylic acid; UFH, unfractionated heparin; SBP, systolic blood pressure; DBP, diastolic blood pressure.

exclusion criterion elevated the bleeding risk up to 2.9-fold (95\% CI, 2.2-3.9). ${ }^{1}$ This information is highly important when considering the use of new OACs.

In contrast to the multiple coagulation-factor targets of VKA, the new OACs inhibit a targeted activated coagulation factor, that is, thrombin (dabigatran) or factor Xa (FXa) (rivaroxaban and apixaban). VKAs have many limitations, and their therapeutic range may be achievable only $60 \%$ of the time, even in highly controlled and selected study populations. ${ }^{2}$ Anticoagulation therapy with direct thrombin inhibitors (DTIs) or FXa inhibitors (DFXaIs) seems to show similar or even better efficacy and safety than do lowmolecular-weight heparins (LMWHs) and VKAs, but thus far only in highly selected patient populations. ${ }^{3-11}$

The new OACs are emerging in clinical practice for prophylaxis of stroke in AF and for prophylaxis and treatment of VTE. Clinical experiences regarding new OACs among nonselected patient populations are scarce, because the initial official indications included only short-term prophylaxis after elective knee or hip replacement surgery. Stroke prevention in AF will become a new indication for dabigatran, rivaroxaban, and apixaban, where dabigatran is the first newcomer. Hence, clinicians should become acquainted with the specific safety characteristics of these drugs and of those patients most likely to gain the greatest long-term benefit from the new OACs.

\section{Bleeding Risk-Related Exclusion Criteria}

Studies on new OACs for VTE treatment or stroke prevention in AF (-Tables 1 and 2 ) excluded patients with another indication for VKA, or having active liver (or alanine aminotransferase $>2$ to 3 times upper normal limit) or kidney disease (an estimated creatinine clearance $<30 \mathrm{~mL} / \mathrm{min}$;
$<25 \mathrm{~mL} / \mathrm{min}$ in Aristotle trial). ${ }^{6-9,12,13}$ Pregnant or breastfeeding women were also excluded. Use of fibrinolytic agents was excluded in VTE trials. Recent stroke (within 7 to 14 days and severe disabling stroke within the previous 6 months for the RE-LY study) was an exclusion criterion in AF trials.

Assessment of the bleeding risk-related exclusion criteria on admission was subjective, with criteria varying between studies ( - Tables $\mathbf{1}$ and $\mathbf{2}$ ). One could argue that possibly 25 to $40 \%$ of the patients in clinical practice would have been excluded from these studies based on study trial-defined exclusion criteria. ${ }^{1}$ Avoidance of bleeding complications is extremely important; each bleeding event during antithrombotic treatments contributes to highly increased mortality. ${ }^{14-}$

16 One particular challenge is that patients requiring anticoagulation often have simultaneous risk factors predisposing them to both bleeds and thrombosis, that is, diabetes, advanced age, and hypertension according to $\mathrm{CHA}_{2} \mathrm{DS}_{2}$-VASC score (Congestive heart failure, Hypertension, Age $>=75$ [doubled], Diabetes, Stroke [doubled], Vascular disease, Age 65 to 74, and Sex Category [female]) and HAS-BLED score (Hypertension, Abnormal renal/liver function, Stroke, Bleeding history or predisposition, Labile INR, Elderly, Drugs/alcohol concomitantly). ${ }^{17,18}$

Anticoagulation is necessary in various clinical situations. Need for active assessment among patients on new OACs can develop in various scenarios: (1) short-term thromboprophylaxis during acute illness, (2) after orthopedic or other surgery, (3) long-term secondary prophylaxis for stroke or VTE and onset of new disease or need of elective or emergency invasive intervention, (4) cancer patients with simultaneous bleeding and thrombosis risks, or (5) bleeding complication or severe trauma while on new OACs. 
Table 2 Bleeding Risk-Related Exclusion Criteria in Studies Comparing New OACs with Warfarin for Stroke Prevention in AF

\begin{tabular}{|c|c|}
\hline Study, Mean Duration & Bleeding Risk-Related Exclusion Criteria \\
\hline $\begin{array}{l}\text { Dabigatran } \\
\text { (RE-LY) } \\
\text { Median duration, } 2 \text { y } \\
(n=18,113)\end{array}$ & $\begin{array}{l}\text { Conditions associated with increased risk for bleeding: } \\
\text { - Major surgery within the previous month } \\
\text { - Planned surgery or intervention within the following } 3 \text { mo } \\
\text { - History of intracranial, intraocular, spinal, retroperitoneal, or atraumatic } \\
\text { intra-articular bleeding } \\
\text { - Gastrointestinal hemorrhage within the past year } \\
\text { - Symptomatic or endoscopically documented gastroduodenal ulcer disease in the past } 30 \mathrm{~d} \\
\text { - Hemorrhagic disorder or bleeding diathesis } \\
\text { - Fibrinolytic agents within } 48 \mathrm{~h} \\
\text { - Uncontrolled hypertension }(\mathrm{SBP}>180 \mathrm{~mm} \mathrm{Hg} \text { and/or DBP }>100 \mathrm{~mm} \mathrm{Hg}) \\
\text { - Recent malignancy or radiation therapy }(<6 \mathrm{mo}) \text { and expected survival }<3 \text { y } \\
\text { Hemoglobin }<100 \mathrm{~g} / \mathrm{L} \\
\text { Platelet count }<100 \times 10^{9} / \mathrm{L} \\
\text { ASA }>100 \mathrm{mg} / \mathrm{d}\end{array}$ \\
\hline $\begin{array}{l}\text { Rivaroxaban }{ }^{12, \mathrm{~b}} \\
\text { (Rocket-AF) } \\
\text { Median duration, } 589 \text { days } \\
(n=14,264)\end{array}$ & $\begin{array}{l}\text { Active internal bleeding } \\
\text { History of, or condition associated with, increased bleeding risk, including: } \\
\text { - Major surgical procedure or trauma within } 30 \mathrm{~d} \\
\text { - Clinically significant gastrointestinal bleeding within } 6 \text { mo } \\
\text { - History of intracranial, intraocular, spinal, or atraumatic intra-articular bleeding } \\
\text { - Chronic hemorrhagic disorder } \\
\text { - Known intracranial neoplasm, arteriovenous malformation, or aneurysm } \\
\text { - Planned invasive procedure with potential for uncontrolled bleeding, } \\
\text { including major surgery } \\
\text { Hemoglobin <100 g/L } \\
\text { ASA > } 100 \mathrm{mg} / \mathrm{d} \\
\text { ASA in combination with thenopyridines within } 5 \mathrm{~d} \\
\text { Intravenous antiplatelet agents within } 5 \mathrm{~d} \\
\text { Fibrinolytics within } 10 \mathrm{~d} \\
\text { Anticipated need for long-term treatment with NSAID }\end{array}$ \\
\hline $\begin{array}{l}\text { Apixaban }^{13} \\
\text { (Aristotle) } \\
\text { Completed May } 2011 \\
(n=18,201)\end{array}$ & $\begin{array}{l}\text { Increased bleeding risk believed to be a contraindication to OAC } \\
\text { (e.g., previous intracranial hemorrhage) } \\
\text { Persistent uncontrolled hypertension (SBP }>180 \mathrm{~mm} \mathrm{Hg} \text { or DBP }>100 \mathrm{~mm} \mathrm{Hg} \text { ) } \\
\text { Planned major surgery } \\
\text { Inability to comply with INR monitoring } \\
\text { Hemoglobin }<90 \mathrm{~g} / \mathrm{L} \\
\text { Platelet count }<100 \times 10^{9} / \mathrm{L} \\
\text { ASA }>165 \mathrm{mg} / \mathrm{d} \\
\text { Coadministration of aspirin and a thienopyridine }\end{array}$ \\
\hline
\end{tabular}

a Online supplementary appendix RE-LY exclusion criteria http://www.nejm.org/doi/suppl/10.1056/NEJMoa0905561/suppl_file/nejm_connolly_1139sa1.pdf

bhttps://www.dtmi.duke.edu/website-administration/files/ROCKET-AF-LBCT-FINAL.ppt/view

OAC, oral anticoagulant; AF, atrial fibrillation; SBP, systolic blood pressure; DBP, diastolic blood pressure; ASA, acetylsalicylic acid; NSAID, nonsteroidal anti-inflammatory drug; INR, international normalized ratio.

\section{Short-Term Thromboprophylaxis}

Short-term thromboprophylaxis is obviously less problematic than long-term use of the new OACs. Acute infection, transient renal or hepatic insufficiency, or postoperative or other inflammation or problems in drug absorbance can all modify coagulation. The effects of new OACs on measurements of fibrinogen and of other activity-based coagulation factors should thus be known in advance to ensure appropriate interpretation of test results. ${ }^{19-21}$

\section{Long-Term Treatment: Lessons from the Last Decade}

At present, data on the new OACs are based on clinical trials with selected patient populations; no postmarketing safety analyses are yet available. In the RE-LY study, having the longest longitudinal follow-up of patients on new OACs, the AF patients were on dabigatran for only a median 2 years. ${ }^{6}$ Thus, its long-term safety remains unknown during lifelong indications for OAC. Side effects are more common in unselected populations due to aging and comorbidities, such as cancer and renal or liver impairment. Even recently many new drugs with great expectations have been withdrawn from the market due to unexpected off-target side effects, such as hepatotoxicity (the anticoagulant melagatran/ximelagatran and anti-inflammatory nimesulide), cardiotoxicity (the anti-inflammatory rofecoxib and valdecoxib and the antiobesity drug sibutramine), depression (the antiobesity drug rimonabant), or progressive multifocal leukoencephalopathy (the psoriasis drug efalizumab). ${ }^{22-27}$ In addition, dronedarone was newly marketed 
Table 3 Patient Monitoring to Rule Out Ongoing Bleeding or Tendency to Bleed While on an OAC

\begin{tabular}{|l|l|}
\hline Clinical Risk Assessment & Rationale/Aim \\
\hline Blood cell count & Exclusion of anemia and/or thrombocytopenia \\
\hline Renal function & Exclusion of risk for drug accumulation (e.g., $\mathrm{CL}_{\mathrm{CR}}$ ) \\
\hline Liver function & Exclusion of liver impairment (e.g., PT, ALT, prealbumin) \\
\hline Blood pressure & Normotension \\
\hline $\begin{array}{l}\text { Comedications } \\
\text { Head or other severe trauma } \\
\text { (especially in elderly patients) }\end{array}$ & $\begin{array}{l}\text { Evaluation of possible interactions or additive effects } \\
\text { (platelet inhibitors, NSAID, SSRI, SNRI, large doses of omega-3 fatty acid) }\end{array}$ \\
\hline Elective surgery & Emergency assessment and follow-up \\
\hline Active cancer & Peri- and postoperative management plan \\
\hline HAS-BLED ${ }^{18}$ or other bleeding score & Evaluation of benefit-risk ratio \\
\hline
\end{tabular}

$\mathrm{OAC}$, oral anticoagulant; $\mathrm{CL}_{\mathrm{CR}}$, estimated creatinine clearance; PT, prothrombin time; ALT, alanine aminotransferase; NSAID, nonsteroidal antiinflammatory drugs; SSRI, serotonin selective reuptake inhibitors; SNRI, selective noradrenaline reuptake inhibitors; HAS-BLED score (Hypertension, Abnormal renal/liver function, Stroke, Bleeding history or predisposition, Labile INR, Elderly, Drugs/alcohol concomitantly).

for rate control in AF without the need for laboratory checks, but soon after its use in real-world populations, new recommendations were given for monthly laboratory tests during the first 6 months, to survey for liver toxicity. ${ }^{28}$ The coadministration of new OACs and dronedarone in AF patients cannot be recommended.

Long-term use of DTIs and DFXaIs needs further attention as both thrombin and FXa have other key roles beyond coagulation, such as regulatory functions during inflammation and cell renewal. ${ }^{29-31}$ At present caution seems a better approach toward the new OACs than an overoptimistic reliance on, or extrapolation of, the data available.

\section{Need for Elective or Emergency Intervention}

Discontinuation of the new OACs before elective surgery due to obvious bleeding risk is the recommendation, based on pharmacological data. ${ }^{32-34}$ Especially among the elderly with slow drug elimination or for those undergoing surgical procedures requiring intact hemostasis (e.g., neurosurgery), laboratory assessment must demonstrate a reverted anticoagulant effect. In the clinical trials, recent or planned elective surgery was one exclusion criterion. In emergency situations, diagnostic and therapeutic support of sufficient hemostatic capacity may at best prevent excessive bleeding and guide reversal strategies in the absence of specific antidotes. Finally, although discontinuation of OACs would allow performance of hemostatically safe interventions, it remains uncertain how these patients will meet the thrombogenic challenge of major procedures, such as orthopedic or vascular surgery. Will a rebound of thrombin generation ensue during early perioperative hours before thromboprophylaxis has been implemented? Or will the anticoagulant, having an extravascular distribution, impair the extrinsic pathway and tissue support of coagulation?

\section{Bleeding While on New OACs}

In addition to the current lack of specific antidotes, information is only suggestive without clinical evidence as to the roles of blood products, hemostatic (prothrombin complex concentrates [PCC], activated PCC, or recombinant FVIIa), or antifibrinolytic agents in management of bleeds associated with the new OACs. ${ }^{32,35}$ The rapid evolution of the antidote is thus most welcome. Only dabigatran can be eliminated by dialysis, in contrast to rivaroxaban and apixaban-which due to their binding to proteins cannot. ${ }^{32,34}$ Charcoal may be a possibility, at less than 2 hours after the last dose of dabigatran. ${ }^{32}$ Completely unknown is the management and outcome in association with surgery due to a severe bleed, such as aortic aneurysm rupture or gastric ulcer. Avoiding bleeding complications is vital; some may be avoided with targeted patient selection and surveillance (exclusion of developing concomitant anemia, thrombocytopenia, or renal or liver failure; - Table 3 ) and individualized choice of anticoagulant.

Comparison of the data available on OACs is difficult due to the lack of uniform criteria for evaluation of bleeding complications. ${ }^{36}$ In future, the use of International Society on Thrombosis and Haemostasis definitions will harmonize this evaluation of the current and newer anticoagulants. ${ }^{37}$ For postmarketing surveillance, we recommend the documentation of bleeding complications in patient records using International Classification of Diseases (ICD-10-CM): D68.32 stands for hemorrhagic disorder due to extrinsic circulating anticoagulants.

\section{Assessment of Anticoagulation Effect}

Routine laboratory monitoring of the anticoagulation effect is unnecessary, because DTIs and DFXaIs have low interindividual variability, relatively short half-lives ( $<17$ hours), and 
Table 4 Special Situations Making Assessment of Anticoagulant Effects of New OACs Necessary

\begin{tabular}{|l|l|}
\hline Case Scenarios & Underlying Causes \\
\hline $\begin{array}{l}\text { Thrombosis, e.g., unconscious patient with a } \\
\text { stroke }\end{array}$ & $\begin{array}{l}\text { Adequate anticoagulant effect } \\
\text { Compliance and safety of possible thrombolysis }\end{array}$ \\
\hline Bleeding complication & Spontaneous, due to a tissue defect \\
\cline { 2 - 2 } & Accumulation of drug (e.g., kidney dysfunction) \\
\cline { 2 - 2 } & Overdose of drug (accidental or deliberate) \\
\hline Severe trauma & Ensuring sufficient hemostasis during surgery and recovery \\
\hline Elderly patients & Risk of accumulation and increased vulnerability to bleeding risk \\
\hline Progressive renal failure & Kidney-dependent metabolism; risk for drug accumulation \\
\hline Progressive liver insufficiency & Increased risk for bleeding complications \\
\hline Main drug-drug interactions & $\begin{array}{l}\text { P-glycoprotein-mediated pharmacokinetics (dabigatran) } \\
\text { CYP3A4 or P-glycoprotein-mediated pharmacokinetics } \\
\text { (rivaroxaban and apixaban) }\end{array}$ \\
\hline
\end{tabular}

OACs, oral anticoagulants; CYP3A4, cytochrome P450 3A4.

only a few drug-drug interactions based on pharmacokinetic and pharmacodynamic studies. ${ }^{11,34,38-40}$ Criteria for appropriate laboratory monitoring require intra- and interindividual variability of the drug, suitable assay methods, established therapeutic ranges, and proven value of therapeutic drug monitoring. ${ }^{41}$ A recent debate on laboratory monitoring of new OACs reached a consensus: In unselected patient populations, measurement of the anticoagulation effect after a therapeutic dose may be necessary in special circumstances. $^{41,42}$

\section{Special Circumstances and Patient Monitoring}

Special circumstances in which measurement of pharmacodynamic effects of the new OACs could aid in clinical judgment are listed in -Table 4. For planning thrombolysis, emergency evaluation of an unconscious patient with a stroke who is on a new OAC would benefit from a qualitative test of compliance and bleeding risks. In turn, a quantitative method could distinguish between a spontaneous bleed due to a tissue defect versus an excessive drug concentration due to accumulation or overdose. Certain individual clinical features initially and during surveillance must be recognized to avoid complications and difficulties during emergency situations (-Table 3). Mild or moderate renal failure and transient renal impairment are common, especially among elderly and medical patients. Dabigatran mainly ( $\approx 80 \%)$ and rivaroxaban (up to $66 \%$ ) are excreted by the kidneys; severe renal insufficiency is thus a contraindication (estimated creatinine clearance $\left[\mathrm{CL}_{\mathrm{CR}}\right]<30 \mathrm{~mL} / \mathrm{min}$ ) due to risk of drug accumulation. ${ }^{34,43}$ Apixaban is metabolized by multiple elimination pathways but only $25 \%$ of it via the kidneys. ${ }^{40}$ The multiple elimination pathways of apixaban may present an advantage for patients with kidney or liver insufficiency, but more data are necessary. Patient education is important to ensure early contact with physicians on occurrence of any complication such as melena, hematuria, extensive menstruation, unexplained fatigue, or head or blunt trauma. The same applies to disrupted compliance, such as with vomiting, diarrhea, or other problems. Patient monitoring must improve the safety and effectiveness of treatment under special circumstances, and this requires further experience.

\section{Timing of Blood Sampling and Laboratory Measurements}

Preanalytical factors such as patient-related issues (a stable vs. postoperative or bleeding patient) and timing of the last dose before blood collection all impact the interpretation of coagulation measures. The relatively short half-lives of the new OACs require defined timing of sampling related to their administration. Peak levels occur within 1.25 to 3 hours after administration with dabigatran, 2 to 4 hours with rivaroxaban, and 1 to 3 hours with apixaban. ${ }^{11,34}$ Stabilization of a drug's effect may be impaired shortly after surgery or during gastroenteric diseases. Mean half-lives of the new OACs are: 12 to 14 hours for dabigatran, 7 to 13 hours for rivaroxaban, and 8 to 13 hours for apixaban. ${ }^{11,34}$ Proper interpretation of the coagulation test results requires knowledge of the time of sampling after drug administration, dosage, and patient's clinical status. This applies also to activity-based thrombophilia testing (e.g., activated protein C-resistance methods). ${ }^{20,21}$

\section{Global Coagulation versus Substance-Specific Tests}

Options for measurement of the pharmacodynamic effects of the anticoagulants include clotting assays (activated partial thromboplastin time [APTT], prothrombin time [PT], and thrombin time [TT] and its dilution), tests measuring the inhibition of FIla or FXa activity, and functional thrombingeneration tests. ${ }^{44}$ In coagulation-based assays, the reagents and coagulation analyzers are likely to induce significant variation, interfering with comparison between laboratories. $^{20,21}$ Validation of reagent- and method-specific issues is crucial. 
In the absence of data on the new DTIs, data for earlier DTIs offer valuable information on laboratory measurement options. In our studies, in contrast to APTT, both chromogenic methods, the Ecarin chromogenic assay (ECA) and the anti-FIla activity assay (hirudin activity assay) linearly depicted lepirudin ${ }^{45}$ and bivalirudin concentrations (unpublished data) across a wide therapeutic range. Furthermore, the benefit of these chromogenic methods appears to be that they remain intact in the presence of either lupus anticoagulant or warfarin in DTI-spiked plasma. This is in contrast to APTT and prothrombinase-induced clotting time (PiCT ${ }^{\circledR}$, Pefak, Pentapharm, Basel, Switzerland). ${ }^{45-48}$ Diluted TT could also assess therapeutic levels of the DTI lepirudin, even in lupus anticoagulant- and warfarin-containing plasma. ${ }^{45,49}$

\section{Dabigatran}

Plasma concentrations of dabigatran can be reflected in routine coagulation-based tests, because dabigatran inhibits thrombin-mediated formation of fibrin from fibrinogen. PT (or international normalized ratio, INR) is insensitive, and thus cannot exclude a dabigatran effect, whereas INR over 1.5 (Owren PT)/2.0 (Quick PT) may already imply a supratherapeutic dabigatran concentration. ${ }^{21,32,50}$ APTT plateaus out with increasing dabigatran, which predisposes to underestimation of dabigatran concentrations. ${ }^{21,32,38,50}$ Activated clotting time is also insensitive to increasing amounts of dabigatran. ${ }^{32}$

Dabigatran can be best assessed with thrombin-specific TT and ECT-based measures. TT is a highly sensitive indicator of the dabigatran effect ${ }^{32,50}$ and can exclude dabigatran use if normal, whereas quantitative assessment may be achieved by a diluted TT assay such as Hemoclot ${ }^{\circledR}$ thrombin inhibitor assay (Hyphen BioMed, Neuville-sur-Oise, France). ${ }^{32}$ This test needs further evaluation, but a linear correlation between clotting time and dabigatran levels is obtained. ECT, a specificthrombin generation test, linearly depicts plasma concentrations of DTIs, but this assay is cumbersome and needs validation for dabigatran. ${ }^{32,38,50}$ ECA overcomes the limitation of possible prothrombin deficiency when compared with ECT, and it is capable of linear, quantitative measurement of other DTIs, but again no data are available for dabigatran. ${ }^{45,46,48,51,52}$ Neither ECT nor ECA is affected by other OACs. ${ }^{51,52}$

In summary, TT seems to be a sensitive qualitative indicator of dabigatran and can ensure the absence of a relevant dabigatran effect. The traditional APTT is less sensitive and PT is too insensitive, but both should be included in an evaluation of hemostatic status. Diluted thrombin clotting time (Hemoclot) appears promising for quantitive assessment in addition to ECT, but all methods should be validated and standardized for dabigatran.

\section{Rivaroxaban}

Routine coagulation tests are unsuitable for measuring rivaroxaban concentrations. Both PT and APTT, the latter less than the former, are prolonged in a concentration dependent, but insensitive manner as a response to rivaroxaban. ${ }^{53}$ Prolonga- tion may be only transient and its length depends on timing after drug administration. Additionally, both the PT and APTT results varied greatly depending on the reagent used. ${ }^{20,53} \mathrm{PT}$ methods should be calibrated in each laboratory for rivaroxaban and the results displayed as rivaroxaban concentration $(\mu \mathrm{g} / \mathrm{mL}) .^{53}$ Application of the international sensitivity index (ISI)/INR calibration for rivaroxaban seemed feasible in a single-center calibration study, but multicenter studies are necessary to confirm the results. ${ }^{54}$

Incubation times for the HepTest (a clot-based anti-FXa assay) and PiCT test had to be shortened to overcome the paradoxical shortening of clotting times at trough rivaroxaban levels. ${ }^{53}$ PiCT is insensitive to rule out noncompliance despite having a higher sensitivity than PT for rivaroxaban. ${ }^{55,56}$ Rivaroxaban produced a concentration-dependent prolongation of the clotting parameters in thromboelastography and an inhibition of thrombin generation by platelets when measured with endogenous thrombin potential. ${ }^{53,55}$ Rivaroxaban reduced the peak of thrombin generation, and high concentrations almost completely suppressed thrombin generation in a calibrated automated thrombogram..$^{53}$ Chromogenic anti-FXa activity assays calibrated specifically for rivaroxaban are under further evaluation but seem able to assess linearly a wide concentration range of rivaroxaban with high sensitivity. ${ }^{53,55,56}$

\section{Apixaban}

Apixaban only minimally prolonged APTT. ${ }^{57}$ PT was unsuitable for assessment of apixaban due to its relative lack of sensitivity and its assay-dependent variability. ${ }^{58}$ Conversion of PT to INR by using the ISI of thromboplastin reagent further increased measurement variability. In contrast, anti-FXa activity assays linearly covered the relevant plasma concentration ranges of apixaban. Strong and nearly equivalent correlations appeared between plasma apixaban concentrations and anti-FXa activity, with standard curves constructed by either apixaban or even commercial LMWH standards. ${ }^{58}$ Wide availability of the LMWH standards may aid in emergency assessment of apixaban effects, but the methods need to be locally validated.

\section{Conclusions}

Great expectations have arisen for new OACs to permit redirection of the always-limited clinical resources. Even if INR visits can be omitted, outpatient visits cannot. Inevitably, reevaluation of a patient's clinical condition is necessary from time to time while that patient is anticoagulated. This includes ruling out any development of hypertension, anemia, thrombocytopenia, or kidney or liver failure to avoid bleeding complications. Furthermore, launching of the new OACs should be carefully controlled and occur in step-wise fashion. Taking into account the lengthy clinical experience, low costs, and the available antidotes, it would be wise to continue VKAs at least among patients in whom INRs appear stable. However, patients with unstable INRs are not necessary the best candidates for new OACs either, if the underlying reason is poor compliance, particularly as the new OACs have relatively 
short half-lives and omission of doses can be catastrophic. Finally, patients having artificial heart valves with need of anticoagulation should continue with VKA exclusively due to lack of evidence of the new OACs in this indication.

Most likely the safety profile of the new OACs will change or cautions will be added as their use widens; some rare but severe complications can be predicted to occur when exposing hundreds of thousands of "unselected" patients to new drugs that have been trialed in selected patients. All clinicians, but especially general physicians, surgeons, cardiologists, anesthesiologists, and hematologists, when contemplating or facing the side effects of the new OACs, have the responsibility to report expected or unexpected side effects for the sake of future patient safety. Manufacturers are responsible for rapid distribution of easily accessible and timely drug safety data as well as management strategies. Clinicians should develop local and global guidelines for special situations requiring laboratory assessment of new OACs and plan subsequent management and follow-up. We suggest the documenting of any bleeding complications by using the ICD-10 (D68.32) to aid in their systematic analysis.

New OACs are most welcome to overcome the high unmet need of simplified, effective, and safe anticoagulant therapies in comparison with the limitations related to VKAs. In the future, more individualized antithrombotic treatment may become a clinical reality. Individualizing the anticoagulation strategy according to patient-related factors is likely to enhance treatment compliance, safety, and efficacy, but only when the questions related to assessment of the anticoagulant effects, need of antidotes and reversal strategies are answered.

\section{References}

1 Levi M, Hovingh GK, Cannegieter SC, Vermeulen M, Büller HR, Rosendaal FR. Bleeding in patients receiving vitamin $\mathrm{K}$ antagonists who would have been excluded from trials on which the indication for anticoagulation was based. Blood 2008;111(9):4471-4476

2 Ansell J, Hirsh J, Poller L, Bussey H, Jacobson A, Hylek E. The pharmacology and management of the vitamin K antagonists: the seventh ACCP conference on antithrombotic and thrombolytic therapy. Chest 2004;126(3):(Suppl):204S-233S

3 Friedman RJ, Dahl OE, Rosencher N, et al; RE-MOBILIZE, REMODEL, RE-NOVATE Steering Committees. Dabigatran versus enoxaparin for prevention of venous thromboembolism after hip or knee arthroplasty: a pooled analysis of three trials. Thromb Res 2010;126(3):175-182

4 Turun S, Banghua L, Yuan Y, Zhenhui L, Ying N, Jin C. A systematic review of rivaroxaban versus enoxaparin in the prevention of venous thromboembolism after hip or knee replacement. Thromb Res 2011;127(6):525-534

5 Huang J, Cao Y, Liao C, Wu L, Gao F. Apixaban versus enoxaparin in patients with total knee arthroplasty. A meta-analysis of randomised trials. Thromb Haemost 2011;105(2):245-253

6 Connolly SJ, Ezekowitz MD, Yusuf S, et al; RE-LY Steering Committee and Investigators. Dabigatran versus warfarin in patients with atrial fibrillation. N Engl J Med 2009;361(12):1139-1151

7 Schulman S, Kearon C, Kakkar AK, et al; RE-COVER Study Group. Dabigatran versus warfarin in the treatment of acute venous thromboembolism. N Engl J Med 2009;361(24):2342-2352
8 Buller HR, Lensing AW, Prins MH, et al; Einstein-DVT DoseRanging Study investigators. A dose-ranging study evaluating once-daily oral administration of the factor Xa inhibitor rivaroxaban in the treatment of patients with acute symptomatic deep vein thrombosis: the Einstein-DVT Dose-Ranging Study. Blood 2008;112(6):2242-2247

9 Buller H, Deitchman D, Prins M, Segers A; Botticelli Investigators, Writing Committe. Efficacy and safety of the oral direct factor Xa inhibitor apixaban for symptomatic deep vein thrombosis. The Botticelli DVT dose-ranging study. J Thromb Haemost 2008;6 (8):1313-1318

10 Ufer M. Comparative efficacy and safety of the novel oral anticoagulants dabigatran, rivaroxaban and apixaban in preclinical and clinical development. Thromb Haemost 2010;103(3): 572-585

11 Eriksson BI, Quinlan DJ, Eikelboom JW. Novel oral factor Xa and thrombin inhibitors in the management of thromboembolism. Annu Rev Med 2011;62;41-57

12 ROCKET AF Study Investigators. Rivaroxaban-once daily, oral, direct factor Xa inhibition compared with vitamin $\mathrm{K}$ antagonism for prevention of stroke and embolism trial in atrial fibrillation: rationale and design of the ROCKET AF study. Am Heart J 2010;159 (3):340-347, e1

13 Lopes RD, Alexander JH, Al-Khatib SM, et al; ARISTOTLE Investigators. Apixaban for reduction in stroke and other thromboembolic events in atrial fibrillation (ARISTOTLE) trial: design and rationale. Am Heart J 2010;159(3):331-339

14 Eikelboom JW, Mehta SR, Anand SS, Xie C, Fox KA, Yusuf S. Adverse impact of bleeding on prognosis in patients with acute coronary syndromes. Circulation 2006;114(8):774-782

15 Fang MC, Go AS, Chang Y, et al. Death and disability from warfarinassociated intracranial and extracranial hemorrhages. Am J Med 2007;120(8):700-705

16 Guerrouij M, Uppal CS, Alklabi A, Douketis JD. The clinical impact of bleeding during oral anticoagulant therapy: assessment of morbidity, mortality and post-bleed anticoagulant management. J Thromb Thrombolysis 2011;31(4):419-423

17 Camm AJ, Kirchhof P, Lip GY, et al; European Heart Rhythm Association; European Association for Cardio-Thoracic Surgery. Guidelines for the management of atrial fibrillation: the Task Force for the Management of Atrial Fibrillation of the European Society of Cardiology (ESC). Eur Heart J 2010;31(19):2369-2429

18 Lip GY, Frison L, Halperin JL, Lane DA. Comparative validation of a novel risk score for predicting bleeding risk in anticoagulated patients with atrial fibrillation: the HAS-BLED (Hypertension, Abnormal Renal/Liver Function, Stroke, Bleeding History or Predisposition, Labile INR, Elderly, Drugs/Alcohol Concomitantly) score. J Am Coll Cardiol 2011;57(2):173-180

19 Freyburger G, Macouillard G, Labrouche S, Sztark F. Coagulation parameters in patients receiving dabigatran etexilate or rivaroxaban: two observational studies in patients undergoing total hip or total knee replacement. Thromb Res 2011;127(5):457-465

20 Hillarp A, Baghaei F, Fagerberg Blixter I, et al. Effects of the oral, direct factor Xa inhibitor rivaroxaban on commonly used coagulation assays. J Thromb Haemost 2011;9(1):133-139

21 Lindahl TL, Baghaei F, Blixter IF, et al; Expert Group on Coagulation of the External Quality Assurance in Laboratory Medicine in Sweden. Effects of the oral, direct thrombin inhibitor dabigatran on five common coagulation assays. Thromb Haemost 2011;105 (2):371-378

22 Melagatran/ximelagatran: market withdrawal. Prescrire Int 2006;15(83):108

23 Walker SL, Kennedy F, Niamh N, McCormick PA. Nimesulide associated fulminant hepatic failure. Pharmacoepidemiol Drug Saf 2008;17(11):1108-1112

24 Sun SX, Lee KY, Bertram CT, Goldstein JL. Withdrawal of COX-2 selective inhibitors rofecoxib and valdecoxib: impact on NSAID 
and gastroprotective drug prescribing and utilization. Curr Med Res Opin 2007;23(8):1859-1866

25 Williams G. Withdrawal of sibutramine in Europe. BMJ 2010;340; c824

26 Rimonabant: marketing authorization suspended... at last: withdrawal. Half-measures. Prescrire Int 2009;18(100):61

27 Di Lernia V. Progressive multifocal leukoencephalopathy and antipsoriatic drugs: assessing the risk of immunosuppressive treatments. Int J Dermatol 2010;49(6):631-635

28 In brief: FDA warning on dronedarone (Multaq). Med Lett Drugs Ther 2011;53(1359):17

29 Crawley JT, Zanardelli S, Chion CK, Lane DA. The central role of thrombin in hemostasis.J Thromb Haemost 2007;5(Suppl 1):95-101

30 Ansell J. Factor Xa or thrombin: is factor Xa a better target? J Thromb Haemost 2007;5(Suppl 1):60-64

31 Weitz JI. Factor Xa or thrombin: is thrombin a better target? J Thromb Haemost 2007;5(Suppl 1):65-67

32 van Ryn J, Stangier J, Haertter S, et al. Dabigatran etexilate-a novel, reversible, oral direct thrombin inhibitor: interpretation of coagulation assays and reversal of anticoagulant activity. Thromb Haemost 2010;103(6):1116-1127

33 Douketis JD. Pharmacologic properties of the new oral anticoagulants: a clinician-oriented review with a focus on perioperative management. Curr Pharm Des 2010;16(31):3436-3441

34 Eriksson BI, Quinlan DJ, Weitz JI. Comparative pharmacodynamics and pharmacokinetics of oral direct thrombin and factor Xa inhibitors in development. Clin Pharmacokinet 2009;48(1):1-22

35 Crowther MA, Warkentin TE. Bleeding risk and the management of bleeding complications in patients undergoing anticoagulant therapy: focus on new anticoagulant agents. Blood 2008;111 (10):4871-4879

36 Seto AC, Kenyon K, Wittkowsky AK. Discrepancies in identification of major bleeding events in patients taking warfarin. Pharmacotherapy 2008;28(9):1098-1103

37 Schulman S, Kearon C; Subcommittee on Control of Anticoagulation of the Scientific and Standardization Committee of the International Society on Thrombosis and Haemostasis. Definition of major bleeding in clinical investigations of antihemostatic medicinal products in non-surgical patients. J Thromb Haemost 2005;3(4):692-694

38 Stangier J. Clinical pharmacokinetics and pharmacodynamics of the oral direct thrombin inhibitor dabigatran etexilate. Clin Pharmacokinet 2008;47(5):285-295

39 Mueck W, Borris LC, Dahl OE, et al. Population pharmacokinetics and pharmacodynamics of once- and twice-daily rivaroxaban for the prevention of venous thromboembolism in patients undergoing total hip replacement. Thromb Haemost 2008;100(3):453-461

40 Raghavan N, Frost CE, Yu Z, et al. Apixaban metabolism and pharmacokinetics after oral administration to humans. Drug Metab Dispos 2009;37(1):74-81

41 Mismetti P, Laporte S. New oral antithrombotics: a need for laboratory monitoring. For.J Thromb Haemost 2010;8(4):621-626

42 Bounameaux H, Reber G. New oral antithrombotics: a need for laboratory monitoring. Against. J Thromb Haemost 2010;8 (4):627-630

43 Blech S, Ebner T, Ludwig-Schwellinger E, Stangier J, Roth W. The metabolism and disposition of the oral direct thrombin inhibitor, dabigatran, in humans. Drug Metab Dispos 2008;36(2):386-399
44 Dempfle CE, Borggrefe M. Do we need thrombin generation assays for monitoring anticoagulation? Thromb Haemost 2008;100 (2):179-180

45 Salmela B, Joutsi-Korhonen L, Saarela E, Lassila R. Comparison of monitoring methods for lepirudin: impact of warfarin and lupus anticoagulant. Thromb Res 2010;125(6):538-544

46 Siegmund R, Boer K, Poeschel K, Wolf G, Deufel T, Kiehntopf M. Comparison of the ecarin chromogenic assay and different aPTT assays for the measurement of argatroban concentrations in plasma from healthy individuals and from coagulation factor deficient patients. Thromb Res 2008;123(1):159-165

47 Fenyvesi T, Jörg I, Harenberg J. Effect of phenprocoumon on monitoring of lepirudin, argatroban, melagatran and unfractionated heparin with the PiCT method. Pathophysiol Haemost Thromb 2002;32(4):174-179

48 Guy S, Kitchen S, Laidlaw S, Cooper P, Woolley A, Maclean R. The use of ecarin chromogenic assay and prothrombinase induced clotting time in the monitoring of lepirudin for the treatment of heparin-induced thrombocytopenia. Br J Haematol 2008;142 (3):466-468

49 Love JE, Ferrell C, Chandler WL. Monitoring direct thrombin inhibitors with a plasma diluted thrombin time. Thromb Haemost 2007;98(1):234-242

50 Stangier J, Rathgen K, Stähle H, Gansser D, Roth W. The pharmacokinetics, pharmacodynamics and tolerability of dabigatran etexilate, a new oral direct thrombin inhibitor, in healthy male subjects. Br J Clin Pharmacol 2007;64(3):292-303

51 Lange U, Nowak G, Bucha E. Ecarin chromogenic assay-a new method for quantitative determination of direct thrombin inhibitors like hirudin. Pathophysiol Haemost Thromb 2003;33 (4):184-191

52 Lange U, Olschewski A, Nowak G, Bucha E. [Ecarin chromogenic assay: an innovative test for quantitative determination of direct thrombin inhibitors in plasma]. Hamostaseologie 2005;25 (3):293-300

53 Samama MM, Martinoli JL, LeFlem L, et al. Assessment of laboratory assays to measure rivaroxaban-an oral, direct factor Xa inhibitor. Thromb Haemost 2010;103(4):815-825

54 Tripodi A, Chantarangkul V, Guinet C, Samama MM. The International Normalized Ratio calibrated for rivaroxaban has the potential to normalize prothrombin time results for rivaroxaban-treated patients: results of an in vitro study. J Thromb Haemost 2011;9 (1):226-228

55 Graff J, von Hentig N, Misselwitz F, et al. Effects of the oral, direct factor Xa inhibitor rivaroxaban on platelet-induced thrombin generation and prothrombinase activity. J Clin Pharmacol 2007;47(11):1398-1407

56 Lindhoff-Last E, Samama MM, Ortel TL, Weitz JI, Spiro TE. Assays for measuring rivaroxaban: their suitability and limitations. Ther Drug Monit 2010;32(6):673-679

57 Wong PC, Crain EJ, Xin B, et al. Apixaban, an oral, direct and highly selective factor $\mathrm{Xa}$ inhibitor: in vitro, antithrombotic and antihemostatic studies. J Thromb Haemost 2008;6(5): 820-829

58 Barrett YC, Wang Z, Frost C, Shenker A. Clinical laboratory measurement of direct factor Xa inhibitors: anti-Xa assay is preferable to prothrombin time assay. Thromb Haemost 2010; 104(6):1263-1271 